\title{
LENGTH FUNCTIONS ON HYPERCENTRAL GROUPS
}

\author{
by DAVID L. WILKENS
}

(Received 23rd August 1984)

In [6] the structure of any real valued length function on an abelian group $G$ is determined. It is shown there, in Theorem 6.1., that such a length function is an extension of a non-Archimedean length function $l_{1}$ on $N$ by an Archimedean length function $l_{2}$ on $H=G / N$. Any non-Archimedean length function is given by a chain of subgroups, as described in [5], and following from results of Nancy Harrison [2], the length $l_{2}$ is essentially the absolute value function on a subgroup of $R$. In the situation above if $N \neq G$ then $N$ is a subgroup of $G$ whose elements have bounded lengths. In this paper we show that it is an easy consequence of techniques developed in [1] that this result can be extended to hypercentral groups, thus determining the structure of any length function in this case. We point out that the result does not extend to soluble groups. The infinite dihedral group $D_{\infty}$ is soluble. However if $D_{\infty}$ is regarded as a free product of two cyclic groups of order 2 and is given the length function associated with a free product, as described by Lyndon [3], then $N$ is not a subgroup of $D_{\infty}$, and the lengths of its elements are unbounded.

We consider hypercentral groups, that is groups $G$ with the property that every nontrivial epimorphic image of $G$ has a non-trivial centre. For properties of hypercentral groups we refer to Chapter VI of [4], and for definitions and some properties of length functions used in the following theorem, we refer to [1] and [6].

Theorem. If $l$ is a real valued length function on a hypercentral group $G$, then $l$ is an extension of a non-Archimedean length function $l_{1}$ on $N$, the collection of nonArchimedean elements of $G$, by an Archimedean length function $l_{2}$ on $H=G / N$. Moreover there exists $c \geqq 0$ and an embedding $g: H \rightarrow R$ such that $c$ is an upper bound for the lengths of the elements of $N$ and $l_{2}(u)=c+|g(u)|$ for $u \in H, u \neq 1$.

Proof. We consider results established in [1]. Here for $l$ a length function on any group $G$ the maximal $l$-trivializable subgroup $T$ of $G$ is defined by

$$
T=\{a \in G ; l(a x)=l(x) \text { for all } x \notin N\} \text {. }
$$

The subgroup $T \subset N$, and it is shown in Proposition 1.3. that the length function restricted to $T$ can be changed subject only to a bound condition. Two length functions on $G$ are then defined to be equivalent if their maximal trivializable subgroups are identical, and the length functions agree on elements outside this subgroup. By considering the cases where the centre $Z$ of $G$ is or is not contained in $N$, it is shown in Theorem 2.1 of [1] that $l$ is an extension of a non-Archimedean length function $l_{1}$ on $N$ 
by an Archimedean length function $l_{2}$ on $H=G / N$, an abelian group, or $l$ is equivalent to an extension of a non-Archimedean length function $l_{1}$ on $C$, the core of $T$, by a length function $l_{2}$ on $K=G / C$, a group with trivial centre. It is possible above that $C=T=N$, which is the case in (ii) of Theorem 2.1, when the equivalence is, in fact, an equality.

The factor group $K$ above cannot be hypercentral, since it has trivial centre, and hence the group $G$ in this case cannot by hypercentral. If $G$ is assumed to be hypercentral it thus follows that $l$ is an extension of a non-Archimedean length function $l_{1}$ on $N$ by an Archimedean length function $l_{2}$ on the abelian group $H=G / N$.

In Section 3 of [2], Nancy Harrison shows in effect that if $l_{2}$ is an Archimedean length function on an abelian group $H$ then there exists $c \geqq 0$ such that $2 d_{2}\left(u, u^{-1}\right)=$ $2 l_{2}(u)-l_{2}\left(u^{2}\right)=c$ and $2 d_{2}(u, v)=l_{2}(u)+l_{2}(v)-l_{2}\left(u v^{-1}\right) \geqq c$ for all non-trivial $u, v \in H$. Also there is an embedding $g: H \rightarrow R$ such that $l_{2}(u)=c+|g(u)|$. Condition (i) of Proposition 4.1. of [6], must be satisfied by the length functions $l_{1}$ and $l_{2}$, and so $c$ is an upper bound for the lengths of the elements of $N$, completing the proof of the theorem.

\section{REFERENCES}

1. G. R. Chapman and D. L. Wilkens, On length functions, trivializable subgroups and centres of groups, Michigan Math. J. 30 (1983), 165-173.

2. Nancy Harrison, Real length functions in groups, Trans. Amer. Math. Soc. 174 (1972), 77106.

3. Roger C. Lyndon, Length functions in groups, Math. Scand. 12 (1963), 209-234.

4. E. Schenkman, Group Theory (Van Nostrand, 1965).

5. David L. Wilkens, On non-Archimedean lengths in groups, Mathematika 23 (1976), 57-61.

6. David L. Wilkens, Length functions and normal subgroups, J. London Math. Soc. (2) 22 (1980), 439-448.

Department of Mathematics

UNIVERSITY OF BIRMINGHAM

BirminghAM, B15 2TT 